MATEC Web of Conferences 1, 10009 (2012)

DOI: $10.1051 /$ matecconf/20120110009

(C) Owned by the authors, published by EDP Sciences, 2012

\title{
Effect of fast frequency excitation on tower oscillations under turbulent wind flow
}

\author{
I. Kirrou, L. Mokni, M. Belhaq \\ Laboratory of Mechanics, University Hassan II-Casablanca, Morocco
}

\begin{abstract}
In this paper, vibrations of a self-excited tower under turbulent wind flow, in the presence of a fast frequency excitation, are investigated and the corresponding equations of motion are derived. The nonlinear terms in the governing equation due to the simultaneous presence of the self, parametric and external excitation, are considered and the galloping phenomena of the tall cantilever structure is studied. It is demonstrated that fast excitations may shift the frequency response and delays Hopf bifurcation of the tower. For validation of analytical results, comparisons with numerical simulations are given for different kinds of excitations.
\end{abstract}

\section{Introduction}

Considerable efforts have been devoted to the control of linear and nonlinear vibrating structures in general and taller towers in particular. Many of these taller towers are more flexible and aerodynamically sensitive to the natural wind environment. There have been a number of reported failures of towers because of design, construction or maintenance problems and these failures were traced to the neglect of dynamic effects. Consequently, it is important to obtain more information on the dynamic response of tall towers subjected to time-varying wind forces in the natural environment. Wind induces aerodynamic (drag and lift) forces in the tower structures. This is the case of structures subject to steady wind where the aerodynamic force introduces a damping term depending on the wind velocity [1].

In order to point out the main active phenomena such as fold bifurcations, galloping and jump, the analysis of the sources of excitation acting on the structure external, parametric, or self-excited autonomous excitation are dealt [2]. In this context, [3] investigated numerically the effects of turbulence on 2-D coupled galloping treating the wind loading as a stochastic process, pointing out a modification of amplitude oscillations as a function of the integral length scale of turbulence. In [4] a one SDOF system, bearing the simultaneous presence of the self, parametric and external excitations, is considered to study the galloping phenomena of tall cantilever structures. The multiple-scale method (MSM) was used to analyze the effect of primary and secondary resonances on the response of the structure. It is shown that the unsteady component can cause a significant decrease in the wind speed at which large amplitude oscillations occur.

Recently, Luongo and Zulli [5] studied a self-excited tower under turbulent wind flow. The stationary wind is responsible for self-excitation and the turbulent part provides both parametric and external excitations. In this case, the simultaneous occurrence of the three kinds of excitations, namely self, parametric and external excitation is possible.

In this work, attention will be focused on the effects of a high-frequency excitation (HFE) on the dynamic of a self-excited structure under turbulent wind. The structure consists of a tower considered as a linear elastic multistory shear-type frame, modelled through a corresponding homogeneous scheme of continuous shear cantilever and then discretized as one SDOF system via the Galerkin method. For details on the formulation, see [5]. The direct partition of motion (DPM) [9] technique is used to split up the fast and the slow dynamics. Then, the MSM is applied under a specific resonance condition to derive the modulation equations, thereby the frequency-response curves. These curves are used to analyze the effect of the fast excitation on the Hopf bifurcation and on the frequency shift of the tower response.

\section{Equations of motion and slow dynamic}

Consider a SDOF model of a discrete non-dimensional, time periodic system, in the form [5]

$$
\begin{gathered}
\ddot{x}+x+\left(c_{a}(1-\bar{U})-b_{1} u(t)\right) \dot{x}+b_{2} \dot{x}^{2}+ \\
\left(\frac{b_{31}}{\bar{U}}+\frac{b_{32}}{\bar{U}^{2}} u(t)\right) \dot{x}^{3}=\eta_{1} \bar{U} u(t)+\eta_{2} \bar{U}^{2}+Y \cos (v t)
\end{gathered}
$$

where the dot denotes differentiation with respect to the non-dimensional time $t$. Equation (1) contains elastic, viscous and inertial linear terms, as well as quadratic and cubic components in the velocity, generated by the aerodynamic forces. The expressions of coefficients in Eq. (1) are given in Appendix. $Y$ and $v$ are the amplitude and the frequency of the fast external excitation, respectively. The turbulent part is considered as periodic and constituted of its first two frequencies: $u(t)=u_{1} \sin (\Omega t)+u_{2} \sin (2 \Omega t)$, where $u_{1}$ and $u_{2}$ are the amplitudes and $\Omega$ is the fundamental frequency.

In this case, Eq. (1) contains a slow dynamic due to the external excitation and a rapid dynamic produced by the frequency $v$. To investigate the effect of the rapid excitation on the slow dynamic of the tower, we use the method of DPM $[9,12,13]$ which consists in introducing two different time scales, a fast time $T_{0}=v t$ and a slow time $T_{1}=t$, and splitting up $x(t)$ into a slow part $z\left(T_{1}\right)$ and a fast part $\phi\left(T_{0}, T_{1}\right)$ as

$$
x(t)=z\left(T_{1}\right)+\mu \phi\left(T_{0}, T_{1}\right)
$$


where $z$ describes the slow main motions at time-scale of oscillations, $\mu \phi$ stands for an overlay of the fast motions and $\mu$ indicates that $\mu \phi$ is small compared to $z$. Since $v$ is considered as a large parameter, we choose $\mu \equiv v^{-1}$ for convenience. The fast part $\mu \phi$ and its derivatives are assumed to be $2 \pi$-periodic functions of fast time $T_{0}$ with zero mean value with respect to this time, so that $\langle x(t)\rangle=$ $z\left(T_{1}\right)$ where $<>\equiv \frac{1}{2 \pi} \int_{0}^{2 \pi}() d T_{0}$ defines time-averaging operator over one period of the fast excitation with the slow time $T_{1}$ fixed. Introducing $D_{i}^{j} \equiv \frac{\partial^{j}}{\partial^{j} T_{i}}$ yields $\frac{d}{d t}=v D_{0}+D_{1}$, $\frac{d^{2}}{d t^{2}}=v^{2} D_{0}^{2}+2 v D_{0} D_{1}+D_{1}^{2}$ and substituting Eq. (2) into Eq. (1) gives

$$
\begin{aligned}
& \mu^{-1} D_{0}^{2} \phi+D_{1}^{2} z+2 D_{0} D_{1} \phi+D_{1}^{2} \phi+\left(c_{a}(1-\bar{U})-\right. \\
& \left.b_{1} u(t)\right)\left(D_{1} z+D_{0} \phi+\mu D_{1} \phi\right)+z+\mu \phi+b_{2}\left(\left(D_{1} z\right)^{2}+\right. \\
& \left.2 D_{1} z\left(D_{0} \phi+\mu D_{1} \phi\right)+\left(D_{0} \phi\right)^{2}+2 \mu D_{0} \phi D_{1} \phi+\left(\mu D_{1} \phi\right)^{2}\right)+ \\
& \left(\frac{b_{31}}{\bar{U}}+\frac{b_{32}}{\bar{U}^{2}} u(t)\right)\left(\left(D_{1} z\right)^{3}+3\left(D_{1} z\right)^{2}\left(D_{0} \phi+\mu D_{1} \phi\right)+\right. \\
& \left.3\left(D_{1} z\right)\left(D_{0} \phi+\mu D_{1} \phi\right)^{2}+\left(D_{0} \phi+\mu D_{1} \phi\right)^{3}\right)=\eta_{1} \bar{U} u(t)+ \\
& \eta_{2} \bar{U}^{2}+Y \cos (v t)
\end{aligned}
$$

Averaging (3) leads to

$$
\begin{aligned}
& D_{1}^{2} z+\left(c_{a}(1-\bar{U})-b_{1} u(t)\right) D_{1} z+z+b_{2}\left(\left(D_{1} z\right)^{2}+\right. \\
& \left.<\left(D_{0} \phi\right)^{2}>+<\left(\mu D_{1} \phi\right)^{2}>\right)+\left(\frac{b_{31}}{\bar{U}}+\frac{b_{32}}{\bar{U}^{2}} u(t)\right)\left(\left(D_{1} z\right)^{3}+\right. \\
& \left.3 D_{1} z\left(<\left(D_{0} \phi\right)^{2}>+<\left(\mu D_{1} \phi\right)^{2}>\right)\right)=\eta_{1} \bar{U} u(t)+\eta_{2} \bar{U}^{2}(4)
\end{aligned}
$$

Subtracting (4) from (3) yields

$$
\begin{aligned}
& \mu^{-1} D_{0}^{2} \phi+2 D_{0} D_{1} \phi+D_{1}^{2} \phi+\left(c_{a}(1-\bar{U})-b_{1} u(t)\right)\left(D_{0} \phi+\right. \\
& \left.\mu D_{1} \phi\right)+\mu \phi+b_{2}\left(2 D_{1} z\left(D_{0} \phi+\mu D_{1} \phi\right)+\left(D_{0} \phi\right)^{2}-\right. \\
& \left.<\left(D_{0} \phi\right)^{2}>+2 \mu D_{0} \phi D_{1} \phi+\left(\mu D_{1} \phi\right)^{2}-<\left(\mu D_{1} \phi\right)^{2}>\right)+ \\
& \left(\frac{b_{31}}{\bar{U}}+\phi \frac{b_{32}}{\bar{U}^{2}} u(t)\right)\left(3\left(D_{1} z\right)^{2}\left(D_{0}+\mu D_{1} \phi\right)+3 D_{1} z\left(D_{0} \phi\right)^{2}-\right. \\
& 3 D_{1} z<\left(D_{0} \phi\right)^{2}>+6 D_{1} z \mu\left(D_{0} \phi D_{1} \phi\right)+3 D_{1} z\left(\mu D_{1} \phi\right)^{2}- \\
& 3 D_{1} z<\left(\mu D_{1} \phi\right)^{2}>+\left(D_{0} \phi\right)^{3}+3 \mu\left(D_{0} \phi\right)^{2} D_{1} \phi \\
& \left.+3 D_{0} \phi\left(\mu D_{1} \phi\right)^{2}+\mu D_{1} \phi\right)=Y \cos \left(T_{0}\right)
\end{aligned}
$$

Using the so-called inertial approximation [9], i.e. all terms in the left-hand side of Eq. (5), except the first, are ignored, one obtains

$$
\phi=-\mu Y \cos \left(T_{0}\right)
$$

Inserting $\phi$ from Eq. (6) into Eq. (4), using that $\left\langle\cos ^{2} T_{0}\right\rangle=$ $1 / 2$, and neglecting terms of orders greater than three in $z$, give the equation governing the slow dynamic of the motion

$$
\begin{aligned}
& \ddot{z}+\left(c_{a}(1-\bar{U})-b_{1} u(t)+H\left(\frac{b_{31}}{\bar{U}}+\frac{b_{32}}{\bar{U}^{2}} u(t)\right)\right) \dot{z}+z+\phi+ \\
& b_{2} \dot{z}^{2}+\left(\frac{b_{31}}{\bar{U}}+\frac{b_{32}}{\bar{U}^{2}} u(t)\right) \dot{z}^{3}=-G+\eta_{1} \bar{U} u(t)+\eta_{2} \bar{U}^{2}
\end{aligned}
$$

where $H=\frac{3 Y^{2}}{2 v^{2}}$ and $G=-\frac{b_{2} Y^{2}}{2 v^{2}}$.

\section{The multiple scales analysis}

To obtain the frequency response equation, we perform a perturbation method. Introducing a bookkeeping parameter $\varepsilon$ and scaling as $z=\varepsilon^{\frac{1}{2}} z, b_{1}=\varepsilon b_{1}, b_{2}=\varepsilon^{\frac{1}{2}} b_{2}$, $\eta_{1}=\varepsilon^{\frac{3}{2}} \eta_{1}, \eta_{2}=\varepsilon^{\frac{3}{2}} \eta_{2}$ and $H=\varepsilon H$. We assume $\bar{U}=1+\varepsilon V$ and we consider the resonance condition $\Omega=1+\varepsilon \sigma$ where $\sigma$ is a detuning parameter. Using the MSM [2], we seek a two-scale expansion of the solution in the form

$$
z(t)=z_{0}\left(t_{0}, t_{1}\right)+\varepsilon z_{1}\left(t_{0}, t_{1}\right)+O\left(\varepsilon^{2}\right)
$$

where $t_{i}=\varepsilon^{i} t$. In terms of the variables $t_{i}$, the time derivatives become $\frac{d}{d t}=d_{0}+\varepsilon d_{1}+O\left(\varepsilon^{2}\right)$ and $\frac{d^{2}}{d t^{2}}=d_{0}^{2}+2 \varepsilon d_{0} d_{1}+$ $O\left(\varepsilon^{2}\right)$, where $d_{i}^{j}=\frac{\partial^{j}}{\partial^{j} t_{i}}$. Substituting Eq. (8) into Eq. (7), we obtain the following equation and equating coefficients of the same power of $\epsilon$, we obtain

Ordre $\mathrm{O}\left(\varepsilon^{0}\right)$ :

$$
d_{0}^{2} z_{0}+z_{0}=-G
$$

Ordre $\mathrm{O}\left(\varepsilon^{1}\right)$ :

$$
\begin{aligned}
& d_{0}^{2} z_{1}+z_{1}=-2 d_{0} d_{1} z_{0}+\left(c_{a} V+b_{1} u\left(t_{0}\right)-H\left(b_{31}+\right.\right. \\
& \left.\left.b_{32} u\left(t_{0}\right)\right)\right)\left(d_{0} z_{0}\right)-b_{2}\left(d_{0} z_{0}\right)^{2}-\left(b_{31}+b_{32} u\left(t_{0}\right)\right)\left(d_{0} z_{0}\right)^{3}+ \\
& \eta_{1} u\left(t_{0}\right)+\eta_{2}
\end{aligned}
$$

In the case of the principal resonance $(\Omega=1+\epsilon \sigma)$, standard calculations yield the first-order solution

$$
z_{0}=A\left(t_{1}\right) \exp \left(i t_{0}\right)+\bar{A}\left(t_{1}\right) \exp \left(-i t_{0}\right)-G
$$

where $i$ is the imaginary unit and $A$ is an unknown complex amplitude. Then, the turbulent part is written as

$$
u\left(t_{0}, t_{1}\right)=u_{1} \exp \left(i(1+\epsilon \sigma) t_{0}\right)+u_{2} \exp \left(2 i(1+\epsilon \sigma) t_{0}\right)+c c
$$

Substituting Eq. (12) into Eq. (3) and eliminating the secular terms, the modulation equations of the amplitude $a$ and the phase $\gamma$ are given by

$$
\left\{\begin{array}{l}
\dot{a}=a\left(\frac{1}{2}\left(c_{a} V-H b_{31}\right)+\left(H b_{32}-b_{1}\right) \frac{1}{4} u_{2} \sin (2 \phi)\right)+ \\
\left(-\frac{3}{8} b_{31}+\frac{b_{32}}{4} u_{2} \sin (2 \phi)\right) a^{3}-\frac{\eta_{1} u_{1}}{2} \cos (\phi) \\
a \dot{\phi}=a\left[\sigma+H \frac{u_{2}}{4} b_{32} \cos (2 \phi)-\frac{b_{1} u_{2}}{4} \cos (2 \phi)\right]+ \\
\left(\frac{b_{32} u_{2}}{8} \cos (2 \phi)\right) a^{3}+\frac{\eta_{1} u_{1}}{2} \sin (\phi)
\end{array}\right.
$$

\section{Applications and results}

In this section, we analyze the effect of the amplitude of the fast frequency excitation on the vibration of the tower of for different types of turbulent wind flow. The parameter values used for the present study are taken from [5].

\subsection{Non-turbulent wind flow case}

Fixed points of Eq. (13), obtained requiring $\dot{a}=\dot{\phi}=0$, represent periodic oscillations of the system. In the absence of turbulence $\left(u_{1}=u_{2}=0\right)$, only the first equation of Eq. (13) is interesting. Besides the trivial solution, $a=0$, the selfexcitation is responsible for galloping, and given as

$$
\left\{\begin{array}{l}
a=0 \\
a=\sqrt{\frac{4\left(C_{a} V-H b_{31}\right)}{3 b_{31}}}
\end{array}\right.
$$


The behavior of the system, in the absence of turbulence $\left(u_{1}=u_{2}=0\right)$, as given by Eq. (14), for $\sigma=0$, is described in Fig 1. The trivial solution exists everywhere and changes its stability at the bifurcation point. In this figure, we show the effect of the amplitude of the fast frequency excitation on the frequency response of the system. It can be seen from this figure that increasing the amplitude of HFE, the amplitude of the tower response decreases and the Hopf bifurcation point shifts right.

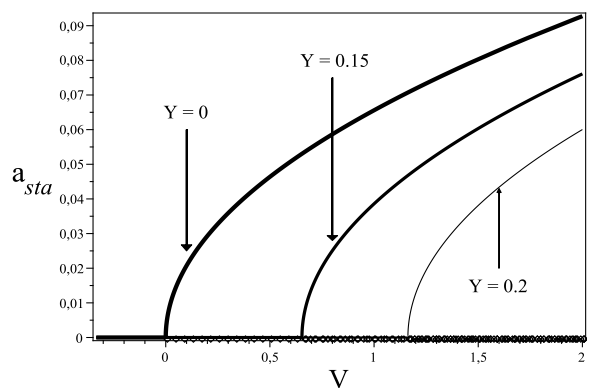

Fig. 1. Equilibrium branches in the absence of turbulent wind, for $v=8$. Solid line: stable; points: unstable.

\subsection{Effects of HFE in the case of turbulent wind}

In this case $\left(u_{1} \neq 0, u_{2}=0\right)$, the modulation equations (13) leads to the following response equation

$$
s_{1}^{2} a^{6}+2 s_{1} s_{2} a^{4}+\left(s_{2}^{2}+\sigma^{2}\right) a^{2}+\left(\frac{\eta_{1} u_{1}}{2}\right)^{2}=0
$$

where $s_{1}=-\frac{3 b_{31}}{8}$ and $s_{2}=\frac{1}{2}\left(C_{a} V-H b_{31}\right)$.

In Figs. 2 and 3, we study the effect of the amplitude of the HFE on the frequency response. Figure 2 illustrates the amplitude of the tower response versus the velocity of the wind $V$, for $u_{1}=0.1$ and $v=8$. These plots indicate that as the amplitude of the HFE increases, the amplitude of tower decreases and shifts right. If $V$ is kept fixed and $\sigma$ is varied, the amplitude of oscillations behave as shown in Fig. 3 , where different values of the amplitude $Y$ are considered. We can see in this figure that, in the absence of HFE $Y=0$, the response curve has three solutions within the interval $(\sigma \in[-0.0001,0.0001])$. It can also be seen that as $Y$ is increased the amplitude of the tower response decreases. Here, only one solution exists. The solid lines correspond to the stable branches, while the points correspond to the unstable ones. To validate the analytical approximation, we integrate (7) numerically using the fourth-order Runge-Kutta method. The comparison demonstrates good agreement between the numerical (circles) and analytical solutions.

\subsection{Effects of HFE in the case of parametric excitation of turbulent wind}

In this case, the system is parametrically excited $\left(u_{1}=\right.$ $0, u_{2} \neq 0$ ), and the corresponding amplitude response equation is written as

$$
\frac{\left(-s_{2} a-s_{1} a^{3}\right)^{2}}{\left(s_{3} a+s_{4} a^{3}\right)^{2}}+\frac{(-\sigma a)^{2}}{\left(s_{3} a+\frac{1}{2} s_{4} a^{3}\right)^{2}}=1
$$

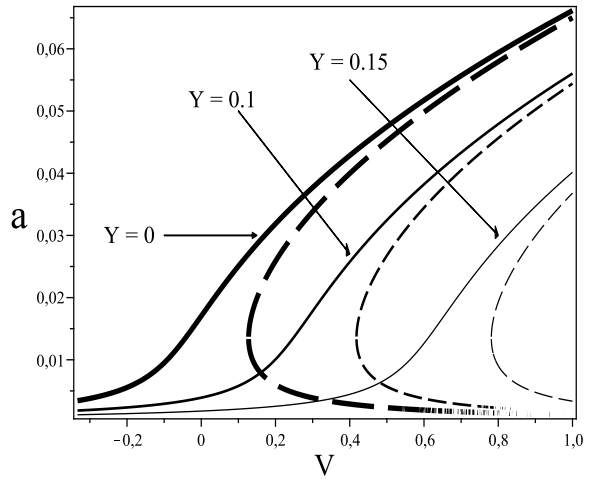

Fig. 2. Amplitude versus velocity of the wind when $\sigma=0, u_{2}=$ $0, u_{1}=0.1$ and $v=8$. Solid line: stable; dashed: unstable.

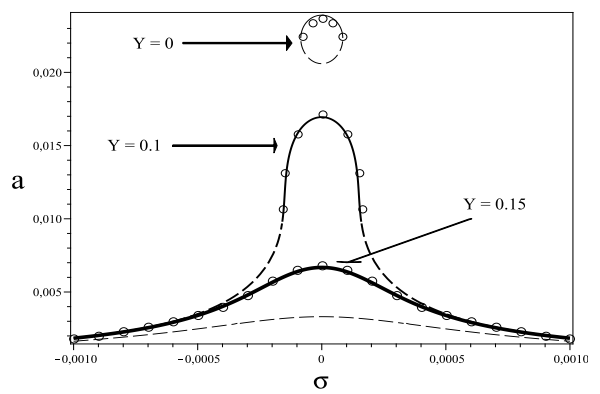

Fig. 3. Amplitude versus $\sigma$ when $V=0.117, u_{1}=0.033, u_{2}=0$ and $v=8$. Solid line: stable; dashed: unstable; circle: numerical simulation.

where $s_{1}=-\frac{3 b_{31}}{8}, s_{2}=\frac{1}{2}\left(C_{a} V-H b_{31}\right), s_{3}=\left(H b_{32}-b_{1}\right) \frac{u_{2}}{4}$ and $s_{4}=b_{32} \frac{u_{2}}{4}$.

Figure 4 shows the amplitude versus the velocity $V$, as given by (16), in the absence of external excitation $\left(u_{1}=\right.$ $\left.0, u_{2} \neq 0\right)$. The solid lines correspond to the stable branches, while the points correspond to the unstable ones. The plots depict the effect of different values of the amplitude $Y$, showing that the amplitude of the response decreases and shifts right as $Y$ increases.

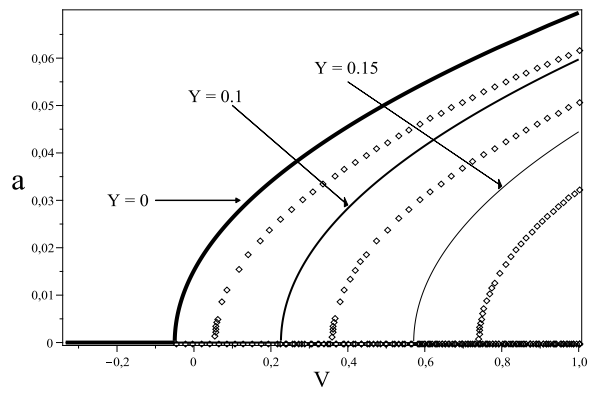

Fig. 4. Amplitude versus $\sigma$ when $\sigma=0, u_{1}=0, u_{2}=0.1$ and $v=8$. Solid line: stable; points: unstable.

\subsection{Effects of HFE in the case of external and parametric excitations of turbulent wind}

In the case where the external and parametric excitations are both present $\left(u_{1} \neq 0, u_{2} \neq 0\right)$, the amplitude frequency response is shows in Fig. 5. The solid lines correspond to 
the stable branches, while the points correspond to the unstable ones. The plots indicate the effect of different values of the amplitude $Y$. It can be seen that the amplitude of the response decreases as $Y$ increases.

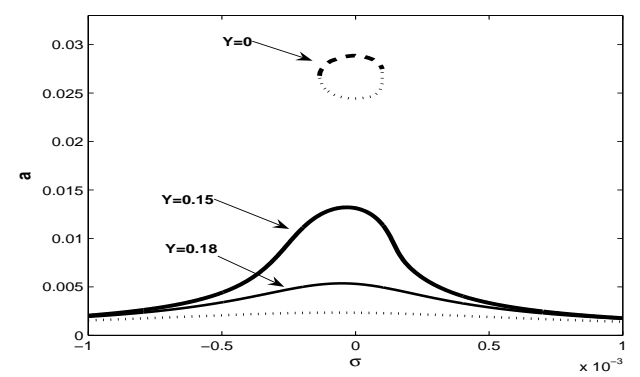

Fig. 5. Amplitude versus $\sigma$ when $V=0.167, u_{1}=0.033, u_{2}=$ 0.033 and $v=8$. Solid line: stable; points: unstable.

\section{Conclusion}

In this work, we have investigated the effect of a fast frequency excitation on the response of a tower under turbulent wind flow near the primary resonance. The method of DPM and the MSM are used to drive a slow dynamic. Results from numerical simulations using Runge-Kutta method are also presented for validation. Different kinds of excitations are analyzed in terms of equilibrium branches of the amplitudes. When the wind is non-turbulent, results show that changing the amplitude of the fast excitation, causes the Hopf bifurcations curve to shift right. When the turbulence produces external excitation only, adding fast frequency excitation causes the amplitude response to decrease significantly and to shift right. The same effects are shown on the Hopf bifurcation curve when the turbulence wind produces either parametric excitation or simultaneous external and parametric excitations.

\section{Appendix}

The expressions of the coefficients of Eq. (1) are $\omega=\pi \frac{\sqrt{3 E I}}{h \ell \sqrt{m}}, c_{a}=\frac{\rho A_{1} b h \ell \bar{U}_{c}}{2 \pi \sqrt{3 E I m}}, b_{1}=c_{a}, b_{2}=-\frac{4 \rho A_{2} b_{1} \ell}{3 \pi m}$ $b_{31}=-\frac{3 \pi \rho A_{3} b \ell \sqrt{3 E I}}{8 h \bar{U}_{c} \sqrt{m^{3}}}, b_{32}=-b_{31}, \eta_{1}=\frac{4 \rho A_{0} b h^{2} \ell \bar{U}_{c}^{2}}{3 \pi^{3} E I}$ $\eta_{2}=\frac{\eta_{1}}{2}, U(t)=\bar{U}+u(t)$

where $\ell$ is the height of the tower, $b$ is the cross-section wide, $E I$ the total stiffness of the single story, $m$ is the mass longitudinal density, $h$ is the inter story height, and $\rho$ is the air mass density.

$A_{i}, i=0, \ldots 3$ are the aerodynamic coefficients for the squared cross-section.

The dimensional critical velocity is given by $\bar{U}_{c}=\frac{4 \pi \xi \sqrt{3 E I m}}{\rho b A_{1} h \ell}$.

Here $\xi$ is the modal damping ratio, depending on both the external and internal dampings:

$\xi=\frac{\eta h^{2}}{24 E I} \omega+\frac{c}{2 m \omega}$

\section{References}

1. R. Clark, A. Modern, Course in Aeroelasticity, fourth ed, Kluwer Academic Publishers, Dordrecht, The Netherlands, 2004.

2. A. H. Nayfeh, B. Balachandran, Applied Nonlinear Dynamics, John Wiley, New York, 1995.

3. Q. S. Li, J. Q. Fang, A. P. Jeary, Evaluation of 2D coupled galloping oscillations of slender structures. Computers and Structures. 66, 513-523, 1998.

4. M. Abdel-Rohman, Effect of unsteady wind flow on galloping of tall prismatic structures, Nonlinear Dyn. 26, 231-252, 2001.

5. A. Luongo, D. Zulli, Parametric, external and selfexcitation of a tower under turbulent wind flow, J. Sound Vib. 330, 3057-3069, 2011.

6. K. Szabelski, J. Warminski, Self-excited system vibrations with parametric and external excitations, J. Sound Vib. 187, 595-607, 1995.

7. M. Eissa, Y. A. Amer, Vibration control of a cantilever beam subject to both external and parametric excitation, Appl. Math. Comput. 152, 611-619, 2004.

8. J. S. Jensen, Non-linear dynamics of the followerloaded double pendulum with added support-excitation, J. Sound Vib. 215, 125-142, 1998.

9. I. I. Blekhman, Vibrational Mechanics, Nonlinear Dynamic Effects, General Approach, Applications. Singapore: World Scientific 2000.

10. D. M. Tcherniak, The influence of fast excitation on a continuous system, J. Sound Vib. 227, 343-360, 1999.

11. M. Hamdi, M. Belhaq, Self-excited vibration control for axially fast excited beam by a time delay state feedback, Chaos Solutions and Fractals. 41, 521-532, 2009.

12. M. Belhaq, A. Fahsi, Hysteresis suppression for primary and subharmonic 3:1 resonances using fast excitation. Nonlinear Dyn. 57, 275-287, 2009.

13. F. Lakrad, M. Belhaq, Suppression of pull-in instability in MEMS using a high-frequency actuation. Commun Nonlinear Sci Numer Simulat. 15, 3640-3646, 2010. 